\title{
A New View of the Universe's Dark Side
}

\section{The Dark Energy Survey Collaboration has updated a model of the} Universe to correctly capture more galaxy measurements.

\section{By Sophia Chen}

$\prod$ he standard cosmological model describes the Universe as expanding at an accelerating rate, with the dominant factors in the expansion rate being two mysterious substances: dark matter and dark energy. To better understand the influences of these dark substances, researchers started mapping the Universe's galaxies, which should provide clues to the distribution of all the Universe's matter. Now, the collaboration behind this work-the Dark Energy Survey (DES) - has built a model that can simultaneously incorporate three types of measurements made from these maps. In doing so, the group establishes new constraints on standard-comological-model parameters that describe the distribution and evolution of dark matter and dark energy [1].

The collaboration used data from DES's first year of observations to track changes in the distribution of regular and dark matter throughout the Universe (see Signing Off from Southern Sky Post). They improved upon their previous analysis by using an updated model for the evolution of the

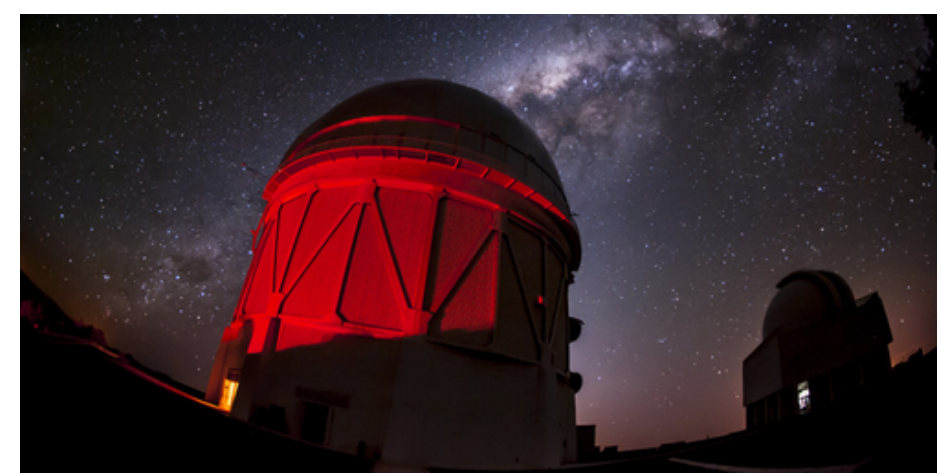

Credit: DES
Universe's matter that captures the relationships between three measurements: the distribution of galaxies, the distribution of galaxies that form clusters, and the distortion of light from faraway galaxies by matter that is closer to Earth.

With this theoretical advance, the collaboration placed constraints on parameters related to the energy density of the Universe's regular and dark matter and to the fluctuations in that density. The revised constraints narrow the allowed values of these parameters by $20 \%$ relative to their previous analysis. Next, the collaboration plans to further refine their dark matter and dark energy measurements by incorporating data from other years of their six-year survey.

Sophia Chen is a freelance science writer based in Columbus, Ohio.

\section{REFERENCES}

1. "Dark Energy Survey year 1 results: Cosmological constraints from cluster abundances, weak lensing, and galaxy correlations," Phys. Rev. Lett. 126, 141301 (2021). 